\title{
Efficient downregulation of ErbB-2 induces TACC1 upregulation in breast cancer cell lines
}

\author{
JINYU XIANG $^{1 *}$, WENSHENG QIU $^{1 *}$, XINGANG WANG $^{2}$, FANG ZHOU $^{1}$, \\ ZHEN WANG ${ }^{1}$, SHIHAI LIU ${ }^{3}$ and LU YUE ${ }^{1}$ \\ Departments of ${ }^{1}$ Oncology, ${ }^{2}$ Galactophore Surgery, and ${ }^{3}$ Central Laboratory, \\ The Affiliated Hospital of the Medical College, Qingdao University, Qingdao 266003, P.R. China
}

Received October 26, 2012; Accepted December 11, 2012

DOI: $10.3892 /$ or.2013.2253

\begin{abstract}
The ErbB-2 gene, whose overexpression is observed in many types of tumors including breast cancer, plays an important role in carcinoma formation. Dysregulation of the human transforming acidic coiled-coil 1 (TACC1) and ErbB-2 genes is thought to be important in the development and progression of breast cancer. However, a putative interaction between ErbB-2 and TACC1 remains undetermined in breast cancer. After infecting BT474 cells with lentiviral-mediated ErbB2-specific shRNA, we detected the expression of ErbB-2 and TACC1 by real-time PCR and western blotting. ErbB-2 mRNA expression was decreased in the Lenti-ShERBB2 infected cells, and western blotting indicated a concordant reduction in ErbB-2 protein. TACC1 expression at the mRNA and protein levels was significantly upregulated by ErbB-2 silencing in BT474 cells. CCK-8 assay indicated that the inhibition of ErbB-2 expression increased the sensitivity of BT474 cells to docetaxel treatment. These findings provide proof and the foundation for the molecular and biological relationships of ErbB-2 and TACC1 in breast cancer.
\end{abstract}

\section{Introduction}

The human epidermal growth factor receptor 2 (HER-2) gene, also called ErbB-2, is one of the most confirmed and commonly amplified genes in breast cancer. Overexpression of ErbB-2 usually results in malignant transformation of cells and accounts for approximately $25 \%$ of all breast cancer cases (1). ErbB-2 is consistently associated with more aggressive tumor phenotypes, a greater likelihood of lymph node involvement, and increased resistance to chemotherapy and endocrine therapy (2-4). The overall survival rate and time of

Correspondence to: Dr Lu Yue, Department of Oncology, The Affiliated Hospital of the Medical College, Qingdao University, 16 Jiangsu Road, Qingdao 266003, P.R. China

E-mail: qdyuelu@163.com

*Contributed equally

Key words: ErbB-2, transforming acidic coiled-coil 1, breast cancer, lentivirus, docetaxel relapse for ErbB-2-positive breast cancer patients are significantly shorter than for patients without ErbB-2 overexpression. Therefore, ErbB-2 is a logical target for breast cancer therapy, and inhibition of ErbB-2 expression leads to the apoptosis of tumor cells (2,5-7). A monoclonal humanized antibody against ErbB-2 (trastuzumab) has been successfully applied in the treatment of ErbB-2-positive breast cancer. ErbB-2 is a cell surface receptor tyrosine kinase (RTK) and becomes internalized upon ligand binding which may trigger a multitude of signaling pathways, such as MAPKs and PI3K. Consequently, ErbB-2 has been shown to trigger signal transduction leading to cell growth and differentiation. Tumors exhibiting ErbB-2 amplification/overexpression have been shown to demonstrate increased aggressiveness and metastatic potential and are associated with decreased overall patient survival $(8,9)$. Therefore, agents targeted to ErbB- 2 can be utilized for breast cancer treatment.

Members of the transforming acidic coiled-coil (TACC) family of proteins have all been implicated in human cancer $(10,11)$. TACC1 is located on a chromosomal region that is amplified in $10-15 \%$ of human breast cancers (12), and is a gene cloned from the breast cancer amplicon 8p11 (11). The TACC1 mRNA is ubiquitously expressed and encodes a protein with an apparent molecular mass of $125 \mathrm{kDa}$; it is cytoplasmic and mainly perinuclear (11). A full-length cDNA of $7758 \mathrm{bp}$ encoding human TACC1 was found in the databases (GenBank, AF049910). Recently, research on TACC1 found that TACC1 is involved in the regulation of interaction between centrosomes and microtubules (13). Overexpression of TACC1 in mouse fibroblasts results in cellular transformation and anchorage independent growth (11). Moreover, research revealed that TACC1 mRNA and/or protein expression is downregulated in various types of tumors, such as breast and lung cancers. Thus, it was speculated that downregulation of TACC1 may alter the control of mRNA homeostasis in polarized cells and may participate in oncogenic processes (14). Ovarian tumors (78.5\%) lack appreciable expression of TACC1, confirming inferences made from published SAGE analysis that TACC1 can be upregulated or lost in cancer $(15,16)$. Another investigator described that the interaction of the TACC1 protein with several protein partners makes it a good candidate to participate in microtubule-associated processes in normal and tumoral cells (17). 
Table I. Four coding regions corresponding to targeted human ErbB-2.

\begin{tabular}{ll}
\hline Oligo name & \multicolumn{1}{c}{ Oligo sequence } \\
\hline shERBB2-1 & 5'-TTGTCAGTATCCAGGCTTTGTACTCGAGTACAAAGCCTGGATACTGACATTTTTC-3' \\
shERBB2-2 & 5'-TCGAGAAAAATGTCAGTATCCAGGCTTTGTACTCGAGTACAAAGCCTGGATACTGACAA-3' \\
& 5'-TGATCACAGGTTACCTATACATCTCGAGATGTATAGGTAACCTGTGATCTTTTTC-3' \\
shERBB2-3 & 5'-TCGAGAAAAAGATCACAGGTTACCTATACATCTCGAGATGTATAGGTAACCTGTGATCA-3' \\
& 5'-TGAATATGTGAACCAGCCAGATCTCGAGATCTGGCTGGTTCACATATTCTTTTTC-3' \\
shERBB2-4 & 5'-TCGAGAAAAAGAATATGTGAACCAGCCAGATCTCGAGATCTGGCTGGTTCACATATTCA-3' \\
& 5'-TGAAGTACACGATGCGGAGACTCTCGAGAGTCTCCGCATCGTGTACTTCTTTTTC-3' \\
Negative sh-control & 5'-TCGAGAAAAAGAAGTACACGATGCGGAGACTCTCGAGAGTCTCCGCATCGTGTACTTCA-3' \\
& 5'-TCGAGAAAAAGCGCGCTTTGTAGGATTCGCTCGAGCGAATCCTACAAAGCGCGCA-3'
\end{tabular}

Interference sequence specific for the ErbB-2 gene, namely pLL2G-shERBB2-1, pLL2G-shERBB2-2, pLL2G-shERBB2-3, pLL2GshERBB2-4 and negative sh-control, respectively.

An opposing view raises the possibility that amplification of TACC1 promotes malignant growth, thereby making TACC1 an attractive candidate gene for promoting tumorigenicity in human breast cancers. Still et al (11) found that deregulation of TACC1 gene expression, directly, through amplification of the entire gene, or through disruption of transcriptional regulatory elements, contributes to the aggressive phenotype noted in breast tumors, and the amplification of TACC1 may contribute to cancer. TACC1 was found to be significantly overexpressed in samples from ER-positive breast cancer patients who relapsed after tamoxifen treatment when compared with samples from patients who did not. TACC1 was found to be upregulated and to act as an oncogene in breast cancer, and TACC1 was correlated with significantly shorter relapse-free survival (18). Another study identified TACC1, NOV and PTTG1 as new candidate genes associated with endocrine therapy resistance in breast cancer (19). TACC1 has also been associated with other genes, such as FGFR1, BRCA1, Aurora B, BRCA2, AZU-1 and WT1 (17,20,21). Accordingly, we hypothesized that TACC1 is highly expressed in some types of tumors, but downregulated in others. Wilson et al used cDNA microarray technology that allows for the simultaneous evaluation of expression, at the mRNA level, of thousands of genes. A limited number of additional genes were found to be upregulated or downregulated by ErbB-2. These included TACC1, which was found to be downregulated both in ErbB2-positive breast cancer cell lines and in ErbB2-overexpressing breast cancers (22). Therefore, in the present study, we investigated the relationship between TACC1 and ErbB-2.

In the present study, we constructed a lentiviral vector mediating RNAi targeting ErbB-2 (pLL2G-shERBB2). The efficiency of pLL2G-shERBB2 plasmids in interfering with ErbB-2 expression was confirmed by western blotting. We used lentiviral infection to knock down ErbB-2 in BT474 cells resulting in reduced expression. After downregulating the expression of ErbB-2, we detected the changes in TACC1 expression at the mRNA and protein levels by real-time PCR and western blotting, respectively. Our results demonstrated changes in the sensitivity of the cells to chemotherapeutic drugs after silencing of the ErbB-2 gene.

\section{Materials and methods}

Cell lines and cell culture. BT474 [ERBB2(+), TACC1(-)] and MCF-7 [ERBB2(-), TACC1(+)] (22) cancer cells were obtained from the Type Culture Collection of the Chinese Academy of Sciences, Shanghai, China. The cells were maintained in RPMI-1640 (Gibco, Carlsbad, CA, USA) supplemented with $10 \%$ FBS (fetal bovine serum) (v/v) and penicillin $(100 \mathrm{U} / \mathrm{ml}) /$ streptomycin $(100 \mathrm{mg} / \mathrm{ml})$ at $37^{\circ} \mathrm{C}$ in $5 \%$ $\mathrm{CO}_{2}, 95 \%$ air.

Construction and production of lentiviral vectors. We designed and cloned an shRNA template into a lentiviral vector. A selfinactivating lentiviral vector containing a GFP reporter and a U6 promoter upstream of the cloning restriction sites (HpaI and XhoI) was used. The introduction of oligonucleotides encoding shRNAs between these restriction sites enables the production of the shRNA in vitro. Four coding regions corresponding to targeted human ERBB2 starting at different positions were used. We constructed four shRNA-ERBB2 lentiviral vectors, namely pLL2G-shERBB2-1, pLL2G-shERBB2-2, pLL2GshERBB2-3 and pLL2G-shERBB2-4, respectively (Table I). Briefly, oligonucleotides were annealed, digested and inserted between the $\mathrm{HpaI}$ and $\mathrm{XhoI}$ restriction sites of the plasmid vector. Some mutations were introduced in the sense sequence of the hairpin structure to facilitate sequence and avoid destruction by bacteria during amplification in the bacterial host. Correct insertions of shRNA cassettes were confirmed by restriction mapping and direct DNA sequencing. We constructed a vector, pLL2G-shERBB2 (Fig. 1), co-infected with recombinant lentiviral vectors into 293T cells using Lipofectamine 2000 (Invitrogen, Carlsbad, CA, USA). To detect the interference effects of the target, the expression of ErbB-2 mRNA and protein was determined using real-time PCR and western blotting, respectively. Recombinant and control lentiviral vectors 


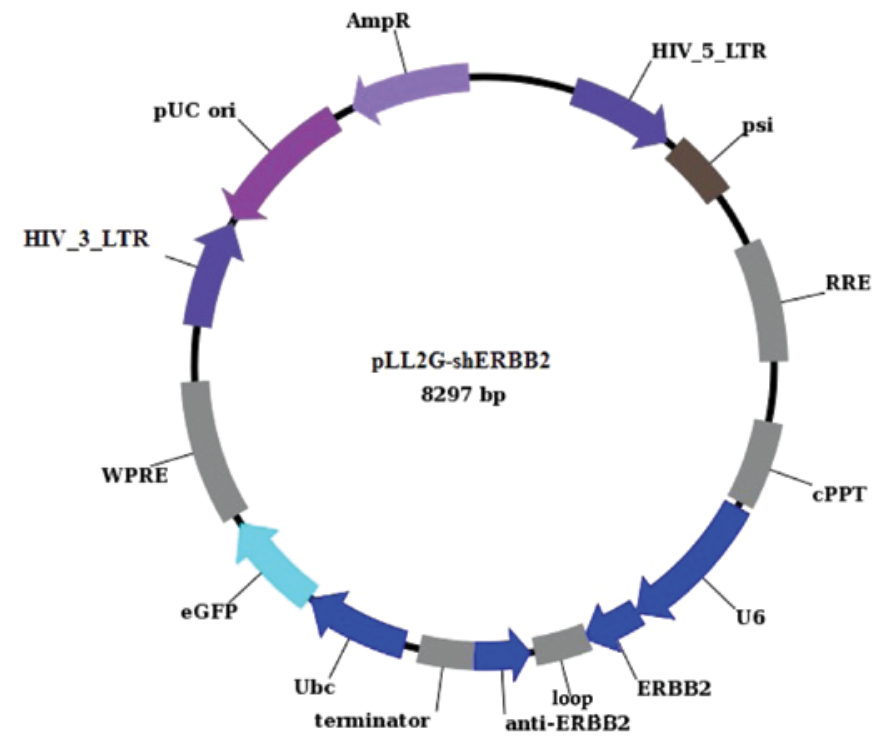

Figure 1. Construction of a lentiviral-mediated shRNA of ErbB-2. Lentiviral vector $\mathrm{pLL2G-shERBB2} \mathrm{containing} \mathrm{an} \mathrm{eGFP} \mathrm{reporter} \mathrm{and} \mathrm{a} \mathrm{U6} \mathrm{promoter}$ upstream of cloning restriction sites ( $\mathrm{HpaI}$ and $\mathrm{XhoI}$ ) to allow the introduction of oligonucleotides encoding shRNAs.

were produced by co-transfecting 293T cells with the lentiviral expression plasmid and packaging plasmids (pLV/helper-SL3, $\mathrm{pLV} /$ helper-SL4 and pLV/helper-SL5). Infectious lentiviral vectors were harvested at $48 \mathrm{~h}$ post-infection, centrifuged to remove cell debris, and filtered through $0.45-\mu \mathrm{m}$ cellulose acetate filters. The infectious titer was determined by hole-bydilution titer assay. The virus titers produced were $\sim 4.9 \times 10^{7}$ $\mathrm{TU} / \mathrm{ml}$

Lentiviral infection. Cells grown in 6-well culture plates (500,000 cells/well) were treated as required. When the cells were $\sim 80 \%$ confluent in complete RPMI-1640 medium, they were infected with the lentiviral constructs. BT474 cells infected with lentiviral-mediated Lenti-shERBB2-eGFP were used as the study cells; cells infected with the negative shRNA (Lenti-sh-control) were used as the negative controls, and cells without infection were considered as the blank controls.

Quantification by real-time PCR. Total RNA was isolated using RNAiso (Takara, Japan). The concentration and purity of RNA were determined using a spectrophotometer. Reverse transcriptase was used to create cDNA for further analyses. Reverse transcriptase and quantitative real-time polymerase chain reaction (RT-PCR) assays were carried out using SYBR Premix Ex Taq II (Perfect Real-Time) (Takara) and real-time PCR amplification equipment. Reverse transcriptase and real-time PCR was carried out according to the instructions provided in the kit. The PCR primers (synthesized by Sangon Biotech, Shanghai, China) used to detect ErbB-2, TACC1 and GAPDH were as follows: ErbB-2, sense strand 5'-CATGTCA TCGTCCTCCAGCAG-3', and antisense strand 5'-TTGACTC TGAATGTCGGCCAA-3', with a product length of $161 \mathrm{bp}$; TACC1, sense strand 5'-TGGTCAGAAATCAGCTGGTGC-3', and antisense strand 5'-GCACAGTGGACCCGTCTTCTT-3', with a product length of $230 \mathrm{bp}$; GAPDH, sense strand 5'-CT GCACCACCAACTGCTTAG-3', and antisense strand 5'-TG
AAGTCAGAGGAGACCACC-3', with a product length of $135 \mathrm{bp}$. The real-time PCR procedure consisted of two steps: one cycle at $95^{\circ} \mathrm{C}$ for $10 \mathrm{sec}$, followed by 40 cycles at $95^{\circ} \mathrm{C}$ for $5 \mathrm{sec}$ and $60^{\circ} \mathrm{C}$ for $20 \mathrm{sec}$. The expression of ErbB-2 was determined by normalization of the threshold cycle of these genes to that of the control housekeeping gene (GAPDH). Data were analyzed using the comparative $\Delta \Delta \mathrm{CT}$ method.

Western blot analysis. Whole-cell proteins in various breast cancer cell lines were isolated. The lysates were centrifuged, and the supernatant was collected and stored at $-80^{\circ} \mathrm{C}$ according to the manufacturer's instructions. Total protein (10 $\mu \mathrm{l}$ ) was loaded per well, separated by $10-15 \%$ SDS-PAGE, and transferred to polyvinylidene difluoride membranes at $60 \mathrm{~V}$ for $1 \mathrm{~h}$ at $4^{\circ} \mathrm{C}$. The membranes were blocked and incubated with primary antibodies (Bioworld Co., USA; diluted 1:1,000 in TBS-A). The membranes were rinsed thrice with $0.1 \%$ Tween 20 -PBS for $30 \mathrm{~min}$. The secondary antibodies (Abcam Co., Cambridge, UK; diluted 1:1,200 in TBS-A) were used with Peroxidase-conjugated Affinipure goat anti-mouse IgG $(1: 8,000)$ and Peroxidase-conjugated Affinipure goat antirabbit $\operatorname{IgG}(1: 8,000)$ for $1 \mathrm{~h}$ at room temperature. The blotted membranes were washed three times with $0.1 \%$ Tween $20-P B S$ for $15 \mathrm{~min}$ and three times with PBS for $15 \mathrm{~min}$. The immunoblots were detected using an electrochemiluminescence kit and exposed to the Vilber Fusion FX5 automatic gel imaging analysis system (Vilber, Marne La Vallée, France).

Cell counting kit-8 (CCK-8) assay. The drug sensitivity assay was carried out using the mitochondrial reduction activity assay. According to the protocol of the CCK-8 assay kit (Dojindo, Kumamoto, Japan), cells grown in 96-well culture plates $(8,000$ cells/well) were treated as required. The experimental group consisted of cells infected with the lentivirus and the control group consisted of non-infected cells. After a 24-h culture, docetaxel (Aventis Pharma, Guildford, UK) was added at the following concentrations: 0.01, 0.05, 0.25, $0.5,0.75,1,5$ and $25 \mu \mathrm{g} / \mathrm{ml}$. Next, cells in each well were incubated with $10 \mu \mathrm{l}$ of CCK-8 at $37^{\circ} \mathrm{C}$ for $4 \mathrm{~h}$. The optical density (OD) for each well was measured at $450 \mathrm{~nm}$ using a microplate reader (Bio-Rad Model 550; Bio-Rad Laboratories, Hercules, CA, USA). CCK-8 experiments were repeated three times on different days. The means and standard deviations of the optical density (OD) of the replicates were calculated for each well. The cell inhibitory rate was calculated according to the following equation: Cell inhibitory rate $=[1-(\mathrm{OD}$ experiment - OD blank)/(OD control - OD blank)] x 100\%. The $50 \%$ inhibition concentration $\left(\mathrm{IC}_{50}\right)$ of the drug was determined by chartography.

Statistical analysis. For all measurements as needed, the statistical significance between groups was assessed by one-way ANOVA based on the homogeneity of variance test (SPSS 13.0, SPSS, Inc., Chicago, IL, USA). P $<0.05$ was considered to indicate a statistically significant difference.

\section{Results}

Selection of the most effective ErbB2-specific shRNA expression vector. Four plasmids containing shERBB2 (pLL2G-shERBB2) 
A

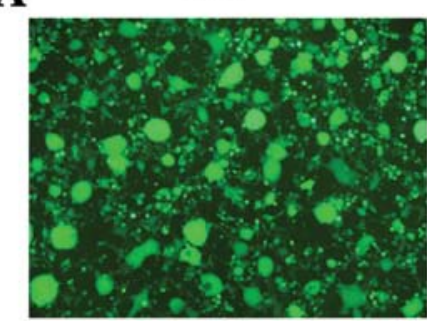

Phase contrast

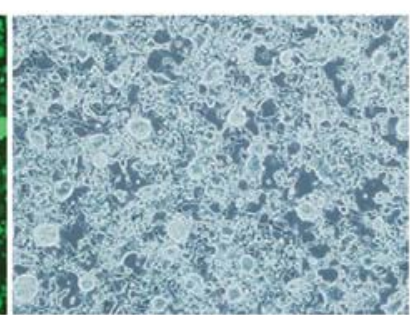

B

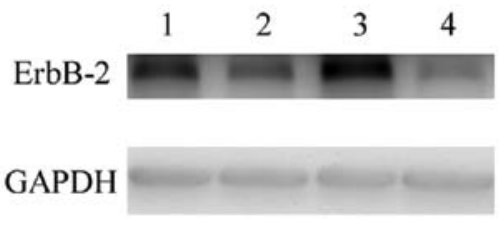

Figure 2. Selection of the most effective ErbB-2-specific shRNA expression vector in 293T cells. (A) Phase contrast and GFP expression under a fluorescence microscope was captured after $48 \mathrm{~h}$ in $293 \mathrm{~T}$ cells. Infection of pLL2G-shERBB2 vector in the 293T cells (original magnification, x200). (B) Protein level of ErbB-2 in the 293 T cells was detected by western blotting. Lanes 1-4, pLL2G-shERBB2-1, -2, -3 and -4, respectively. pLL2G-shERBB2-4 significantly knocked down expression of ErbB-2 at the protein level.
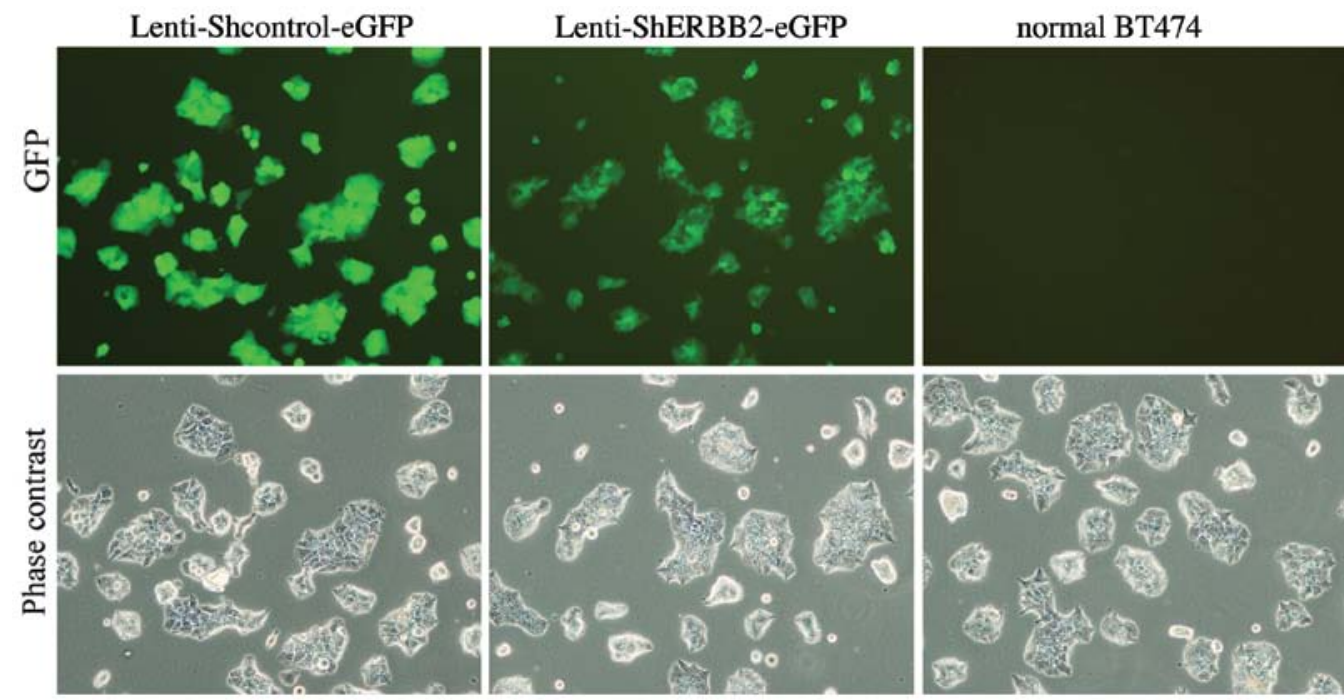

Figure 3. Phase contrast and GFP expression under a fluorescence microscope was captured after $48 \mathrm{~h}$ in the BT474 cells (original magnification, $\mathrm{x} 200$ ). It divided three groups of BT474 cells. Lenti-shcontrol-eGFP groups is added control lentivirus. Lenti-shERBB2-eGFP groups are infected with pLL2G-shERBB2-4. The third group of BT474 cells were without treatment.

were coinfected into $293 \mathrm{~T}$ cells, respectively. GFP expression in the 293T cells was observed under a fluorescence microscope $48 \mathrm{~h}$ after infection with pLL2G-shERBB2. According to the results of the western blotting assay, pLL2G-shERBB2-4 was the most effective lentiviral vector and, thus, was used in the subsequent research (Fig. 2).

Infection efficiency of the viral delivery in vitro. BT474 cells were infected with the lentiviral vector encoding GFP, resulting in GFP expression in the majority of cultured cells. The infection efficiency was assayed by fluorescence microscopy after cells were infected by the lentivirus for 48 h. A robust infection efficiency of $79 \%$ was observed (Fig. 3). The fluorescence gradually weakened and the infected cells began to undergo apoptosis on the fifth day resulting in the gradual decline of the infection rate. BT474 cells that were infected with Lenti-shERBB2-eGFP and non-infected cells co-cultured showed no synergistic growth inhibition effect when treated using the same protocols. We observed that the proliferation of BT474 cells was significantly inhibited after infection with Lenti-shERBB2-eGFP when compared with the Lenti-shcontrol-eGFP and normal BT474 cells $(\mathrm{P}<0.05)$. Realtime PCR and western blot analysis showed that the mRNA and protein expression levels of ErbB-2 were lower in the cells infected with Lenti-shERBB2-eGFP than in the cells infected with the Lenti-shcontrol and the normal BT474 cells $(\mathrm{P}<0.05)$ (Figs. 4 and 5).

mRNA expression of ErbB-2 and TACC1. Following lentiviral infection of BT474 cells, the mRNA expression of ErbB-2 and TACC1 was examined by real-time PCR. A significant difference was noted between the silenced and control cells $(\mathrm{P}<0.05)$, a reduction in the expression of ErbB-2 was noted. The expression of ErbB-2 mRNA in the BT474 cells was successfully knocked down. On the other hand, the expression of ErbB-2 mRNA showed no significant difference between the blank control group and the negative control-shRNA group $(\mathrm{P}>0.05)$. As shown in Fig. 4B, the expression of TACC1 mRNA was affected following ErbB-2 knockdown, with a different expression level noted in the ErbB-2-silenced cells when compared to the control cells. The expression of TACC1 in the ErbB-2-positive cells (BT474) was expressed at a low level. However, after interference of the expression of ErbB-2, expression of TACC1 was upregulated. The result of real-time PCR showed that silencing of the ErbB-2 gene markedly increased the levels of TACC1 mRNA $(\mathrm{P}<0.05)$ (Fig. 4). 
A

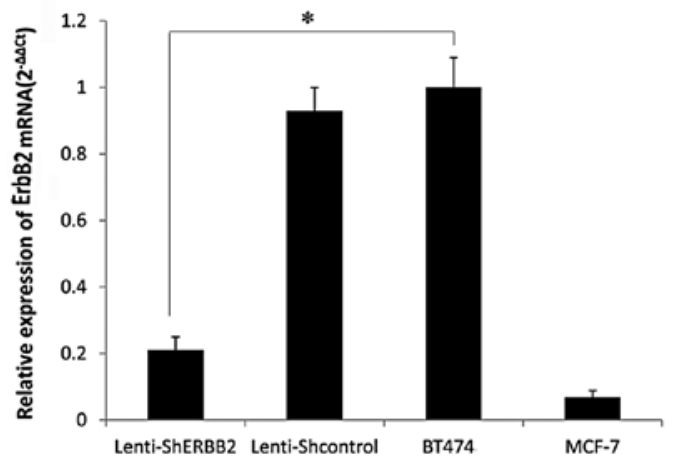

B

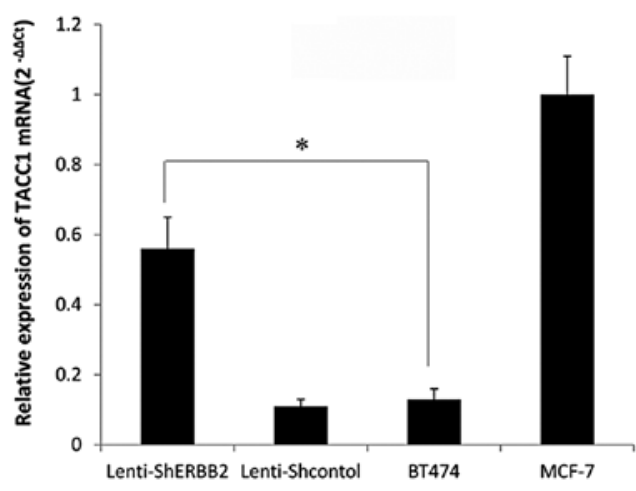

Figure 4. mRNA levels of ErbB-2 and TACC1 detected by real-time PCR after BT474 cells were treated with Lenti-shERBB2 and Lenti-shcontrol ("P<0.05). (A) ErbB2-shRNA-infected cells exhibited significantly lower ErbB-2 mRNA expression $(0.21 \pm 0.01)$ when compared with that of the blank control group $(1 \pm 0.03)$ and negative control-shRNA group (0.93 \pm 0.02$)$. The relative expression of ErbB-2 in MCF-7 cells was $0.069 \pm 0.01$. (B) The relative expression of TACC1 in Lenti-shERBB2 BT474 cells, Lenti-shcontrol BT474 cells, BT474 cells and MCF-7 cells was $0.56 \pm 0.03,0.1 \pm 0.01,0.13 \pm 0.015$ and $1 \pm 0.035$, respectively.
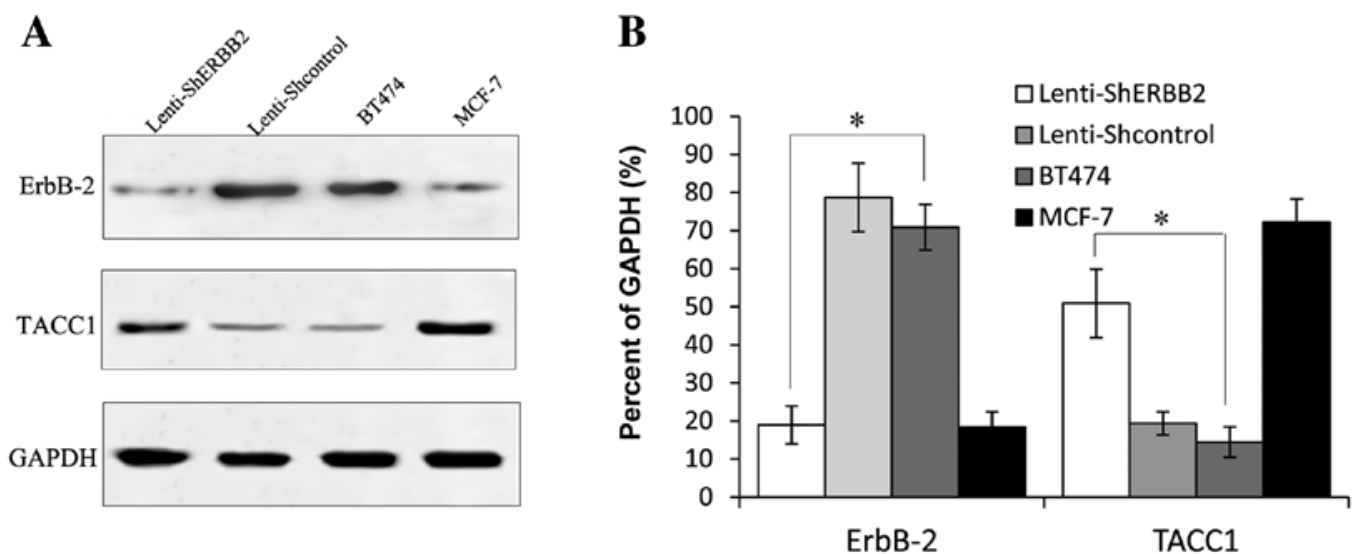

Figure 5. Protein levels of ErbB-2 and TACC1 after BT474 cells were infected with Lenti-shERBB2 and Lenti-shcontrol as detected by western blotting $\left({ }^{*} \mathrm{P}<0.05\right)$. Lenti-shERBB2 significantly knocked down expression of ErbB-2 at the protein level $(\mathrm{P}<0.05)$, compared with Lenti-shcontrol and BT474 cells. After interference of the expression of ErbB-2, TACC1 expression was significantly upregulated $(\mathrm{P}<0.05)$.

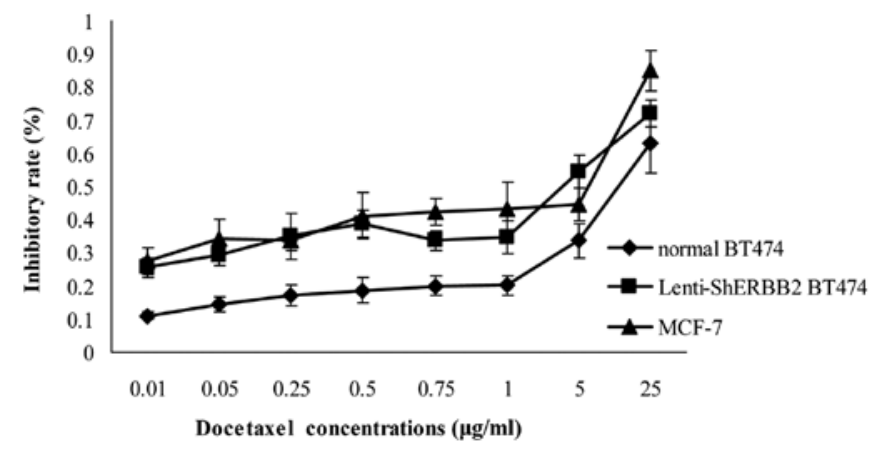

Figure 6. BT474 and MCF-7 cell lines were treated with various concentrations of docetaxel $(0.01,0.05,0.25,0.75,1,5$ and $25 \mu \mathrm{g} / \mathrm{ml})$ for $24 \mathrm{~h}$ for the CCK-8 assay. The inhibitory rates of infected BT474 cells, uninfected BT474 cells and MCF-7 cells are shown. The sensitivity to chemotherapeutic drugs (docetaxel) of BT474 cells increased after ErbB-2 interference, and the difference was statistically significant $(\mathrm{P}<0.05)$. Chemotherapy drug sensitivity of infected BT474 and MCF-7 cells was similar, but the difference was statistically significant.

ErbB-2 and TACC1 protein expression. The protein expression of ErbB-2 and TACC1 was evaluated by western blotting. The gray scale of the stained area was measured under identical conditions. The average optical density for ErbB-2 protein expression in the ErbB2-shRNA-infected cells was lower when compared with the value in the blank control and the negative control-shRNA cells, respectively. This indicated marked reduction in ErbB-2 protein following ErbB2-shRNA infection; the observed differences were significant $(\mathrm{P}<0.05)$ (Fig. 5). Consistent with the above mRNA result, the average optical density for TACC1 protein expression in the BT474, MCF-7, Lenti-shcontrol and Lenti-shERBB-2 cells showed significant differences between the cell groups $(\mathrm{P}<0.05)$ (Fig. 5). TACC1 was overexpressed when the ErbB-2 protein was markedly inhibited by ErbB2-shRNA in BT474 cells. The results were in accordance with the mRNA levels (Fig. 4B).

Effects of Lenti-shERBB2-eGFP on cell sensitivity to docetaxel treatment. Breast cancer cells that overexpress ErbB-2 are more resistant to chemotherapeutic agents such as paclitaxel (Taxol) and docetaxel (Taxotere) than cells that do not overexpress ErbB-2 (23). After inhibiting ErbB-2 expression using Lenti-shERBB2-eGFP infection, the $\mathrm{IC}_{50}$ 
(index of chemotherapy sensitivity to docetaxel) of the LentishERBB2-infected cells was $2.77 \pm 0.04 \mu \mathrm{g} / \mathrm{ml}$. The $\mathrm{IC}_{50}$ of the BT474 cells was $30.01 \pm 0.13 \mu \mathrm{g} / \mathrm{ml}$, and this value for the MCF-7 cells was $1.15 \pm 0.03 \mu \mathrm{g} / \mathrm{ml}$. That is, after ErbB-2 was inhibited, the breast cancer cell sensitivity to chemotherapy improved 10-fold. The result indicated that the supression of ErbB-2 expression inhibits the proliferation of BT474 cells and increases the cell sensitivity to docetaxel treatment; this difference was statistically significant (Fig. 6).

\section{Discussion}

This study confirmed the knockdown of ErbB-2 in BT474 cells by lentiviral infection and that TACC1 exhibited higher expression when ErbB-2 was expressed at a low level. LentishERBB2-eGFP obviously inhibited the expression of ErbB-2 mRNA and protein in BT474 breast cancer cells and also increased the chemotherapy sensitivities to docetaxel in vitro. We also observed that infection with the lentivirus inhibited BT474 cell growth and promoted apoptosis.

ErbB receptors, in particular ErbB-2, play an important role in carcinoma formation, and dysfunction promotes tumorigenesis. Downregulation of ErbB-2 was found to result in an increase in cells in the G1 phase and the induction of apoptosis (24). Overexpression of the ErbB-2 gene has frequently been observed in human tumors, including those of the breast, stomach, lung and oral cavity (25-27). Capable of stable and highly specific silencing of gene expression, shRNAs have been extensively applied to silence abnormal gene expression in the treatment of cancer. Given that ErbB-2 was found to be overexpressed in the BT474 cell line (28), we examined the functional consequence of ErbB-2 silencing in BT474 cells. In the present study, after Lenti-shERBB2 was infected into the BT474 breast cancer cells, ErbB-2 expression was significantly reduced at the mRNA and protein levels. Since ErbB family-mediated signaling plays a critical role in cell growth, survival, adhesion and motility (8), our data suggest that the ErbB-2 gene is a feasible RNAi target for gene silencing. The data described here provide proof that siRNA can be used therapeutically for human cancer. ErbB-2 as well as TACC1 may be referred to as novel cancer therapeutic targets.

It has been documented that TACC1 is downregulated in ErbB-2-positive breast cancer cells and tissues (22). Yet, few studies have examined the functional relationship of TACC1 and ErbB-2 in breast cancer cells. In this study, we investigated the relationship between ErbB-2 and TACC1, specifically in BT474 cells. The expression of TACC1 was examined following Lenti-shERBB2-mediated silencing of the ErbB-2 gene in BT474 cells. We found that silencing of ErbB-2 induced changes in TACC1 expression. Although TACC1 and ErbB-2 both contribute to the progression of certain types of tumors, our data suggest that TACC1 expression may be strictly associated with ErbB-2 amplification in BT474 cells.

In addition to the regulation of expression, TACC1 may be regulated at multiple levels, such as mutations, loss of heterozygosity, promoter methylation, or activation of alternative signaling pathways. Several studies have shown synergistic effects of TACC1 with other genes during tumorigenesis $(17,20,21)$. On the other hand, there is also evidence that TACC1 is involved in drug resistance to chemotherapy and tumor progression (29). Consistent with this hypothesis, research has found that TACC1 overexpression appears to promote paclitaxel-induced cell death (30). It is possible that TACC1 may promote chemosensitivity, resulting from the ability of TACC1 to activate Ras, PI3K and PKB $(29,31)$, whereas the chemosensitivity of TACC1-overexpressing cells may be due to the interaction of TACC1 with microtubules and the mitotic apparatus. In these signaling pathways, TACC1 has been described as transforming (11) and, yet, is downregulated in anthracyclin-treated mammary tumors (14). TACC1 expression results in an increase in both ERK and PKB phosphorylation (32) in mammary tissues. Knuefermann et al (33) delineated a pathway that involves HER2/PI-3K/Akt in mediating multidrug resistance in human breast cancer cells. They found that infection of ErbB-2 in MCF7 breast cancer cells that express HER3 resulted in a phosphoinoside-3 kinase (PI-3K)-dependent activation of Akt, and was associated with an increased resistance of cells to multiple chemotherapeutic agents, including paclitaxel. Regarding PI-3K-dependent Akt activation, while the latter is associated with cell resistance, following the selective inhibition of PI-3K or Akt, cell sensitivity to chemotherapy significantly increased (29). There appears to be a certain correlation between ErbB-2 and TACC1 in PI-3K/Akt-related chemotherapy resistance. If true, overexpression of TACC1 may serve as a useful marker for chemosensitive tumors.

In summary, we demonstrated that ErbB-2 was effectively silenced in BT474 cells via lentiviral infection, providing evidence that the use of lentiviral shRNA can sensitize docetaxel-resistant ErbB-2-overexpressing breast cancer cells to the drug by repressing ErbB-2 expression. This in turn may have important implications for the development of a novel therapy that combines chemotherapy and gene therapy. In addition to new diagnostic and prognostic markers, TACC1 may be a novel target for breast cancer therapy. Future studies are required to examine the molecular and biological relationship of ErbB-2 and TACC1 in breast cancer cells in greater detail.

\section{Acknowledgements}

This study was supported by the Shandong Natural Science Foundation (2009HW024; Y2006C23); the Shandong Excellent Young Scientist Research Award Fund Project (2006BSB14114; BS2010YY013) and the Shandong Tackle Key Problems in Science and Technology (2010GSF10245).

\section{References}

1. Tai W, Qin B and Cheng K: Inhibition of breast cancer cell growth and invasiveness by dual silencing of HER-2 and VEGF. Mol Pharm 7: 543-556, 2010.

2. Bartsch R, Wenzel C, Zielinski CC and Steger GG: HER-2-positive breast cancer: hope beyond trastuzumab. BioDrugs 21: 69-77, 2007.

3. Engel RH and Kaklamani VG: HER2-positive breast cancer: current and future treatment strategies. Drugs 67: 1329-1341, 2007.

4. Dos Santos ML, Gimenes KP, Silva WA Jr and Nagai MA: Transcriptome changes induced by docetaxel in human mammary cell lines expressing different levels of ERBB2. Int J Mol Med 23: 733-743, 2009.

5. Yang G, Cai KQ, Thompson-Lanza JA, Bast RC Jr and Liu J: Inhibition of breast and ovarian tumor growth through multiple signaling pathways by using retrovirus-mediated small interfering RNA against Her-2/neu gene expression. J Biol Chem 279: 4339-4345, 2004. 
6. Faltus T, Yuan J, Zimmer B, et al: Silencing of the HER2/neu gene by siRNA inhibits proliferation and induces apoptosis in HER2/neu-overexpressing breast cancer cells. Neoplasia 6: 786-795, 2004

7. Choudhury A, Charo J, Parapuram SK, et al: Small interfering RNA (siRNA) inhibits the expression of the Her2/neu gene, upregulates HLA class I and induces apoptosis of Her $2 /$ neu positive tumor cell lines. Int J Cancer 108: 71-77, 2004.

8. Menard S, Casalini P, Campiglio M, Pupa SM and Tagliabue E: Role of HER $2 /$ neu in tumor progression and therapy. Cell Mol Life Sci 61: 2965-2978, 2004.

9. Coronado Martin PJ, Fasero Laiz M, Garcia Santos J, Ramirez Mena $\mathrm{M}$ and Vidart Aragon JA: Overexpression and prognostic value of $\mathrm{p} 53$ and HER2/neu proteins in benign ovarian tissue and in ovarian cancer. Med Clin 128: 1-6, 2007 (In Spanish).

10. Still IH, Vince P and Cowell JK: The third member of the transforming acidic coiled coil-containing gene family, TACC 3 , maps in $4 \mathrm{p} 16$, close to translocation breakpoints in multiple myeloma, and is upregulated in various cancer cell lines. Genomics 58 : $165-170,1999$.

11. Still IH, Hamilton M, Vince P, Wolfman A and Cowell JK: Cloning of TACC1, an embryonically expressed, potentially transforming coiled coil containing gene, from the $8 \mathrm{p} 11$ breast cancer amplicon. Oncogene 18: 4032-4038, 1999.

12. Ray ME, Yang ZQ, Albertson D, et al: Genomic and expression analysis of the 8p11-12 amplicon in human breast cancer cell lines. Cancer Res 64: 40-47, 2004.

13. Gergely F, Karlsson C, Still I, Cowell J, Kilmartin J and Raff JW: The TACC domain identifies a family of centrosomal proteins that can interact with microtubules. Proc Natl Acad Sci USA 97: 14352-14357, 2000.

14. Conte N, Charafe-Jauffret E, Delaval B, et al: Carcinogenesis and translational controls: TACC1 is down-regulated in human cancers and associates with mRNA regulators. Oncogene 21: 5619-5630, 2002.

15. Rhodes DR, Barrette TR, Rubin MA, Ghosh D and Chinnaiyan AM: Meta-analysis of microarrays: interstudy validation of gene expression profiles reveals pathway dysregulation in prostate cancer. Cancer Res 62: 4427-4433, 2002.

16. Line A, Slucka Z, Stengrevics A, Li G and Rees RC: Altered splicing pattern of TACC1 mRNA in gastric cancer. Cancer Genet Cytogenet 139: 78-83, 2002.

17. Conte N, Delaval B, Ginestier C, et al: TACC1-chTOGAurora A protein complex in breast cancer. Oncogene 22: 8102-8116, 2003.

18. Nguyen HG, Chinnappan D, Urano T and Ravid K: Mechanism of Aurora-B degradation and its dependency on intact KEN and A-boxes: identification of an aneuploidy-promoting property. Mol Cell Biol 25: 4977-4992, 2005.

19. Ghayad SE, Vendrell JA, Bieche I, et al: Identification of TACC1, NOV, and PTTG1 as new candidate genes associated with endocrine therapy resistance in breast cancer. J Mol Endocrinol 42: 87-103, 2009.
20. Ryser S, Dizin E, Jefford CE, et al: Distinct roles of BARD1 isoforms in mitosis: full-length BARD1 mediates Aurora B degradation, cancer-associated BARD1beta scaffolds Aurora B and BRCA2. Cancer Res 69: 1125-1134, 2009.

21. Devilard E, Bladou F, Ramuz O, et al: FGFR1 and WT1 are markers of human prostate cancer progression. BMC Cancer 6: 272, 2006.

22. Wilson KS, Roberts H, Leek R, Harris AL and Geradts J: Differential gene expression patterns in HER2/neu-positive and -negative breast cancer cell lines and tissues. Am J Pathol 161: 1171-1185, 2002.

23. Ueno NT, Yu D and Hung MC: Chemosensitization of HER-2/neuoverexpressing human breast cancer cells to paclitaxel (Taxol) by adenovirus type 5 E1A. Oncogene 15: 953-960, 1997.

24. Cheng LS, Zha Z, Xi JJ, Jiang B, Liu J and Yao XB: Downregulation of HER 2 by adenovirus-mediated RNA interference and its inhibitory effect on growth of SKBR3 breast cancer cell. Xi Bao Yu Fen Zi Mian Yi Xue Za Zhi 23: 691-695, 2007 (In Chinese)

25. Verri E, Guglielmini P, Puntoni M, et al: HER2/neu oncoprotein overexpression in epithelial ovarian cancer: evaluation of its prevalence and prognostic significance. Clinical study. Oncology 68: 154-161, 2005

26. Nalwoga H, Odida $M$ and Wabinga $H$ : c-erbB-2 oncoprotein over-expression in breast cancer and its relationship to histology and grade in a Ugandan population. East Afr Med J 83: 411-415, 2006.

27. Cornolti G, Ungari M, Morassi ML, et al: Amplification and overexpression of HER2/neu gene and HER2/neu protein in salivary duct carcinoma of the parotid gland. Arch Otolaryngol Head Neck Surg 133: 1031-1036, 2007.

28. Yoon S, Lee MY, Park SW, et al: Up-regulation of acetyl-CoA carboxylase alpha and fatty acid synthase by human epidermal growth factor receptor 2 at the translational level in breast cancer cells. J Biol Chem 282: 26122-26131, 2007.

29. Cully M, Shiu J, Piekorz RP, Muller WJ, Done SJ and Mak TW: Transforming acidic coiled coil 1 promotes transformation and mammary tumorigenesis. Cancer Res 65: 10363-10370, 2005.

30. Lee MJ, Gergely F, Jeffers K, Peak-Chew SY and Raff JW: Msps/XMAP215 interacts with the centrosomal protein D-TACC to regulate microtubule behaviour. Nat Cell Biol 3: 643-649, 2001.

31. Marmor MD, Skaria KB and Yarden Y: Signal transduction and oncogenesis by ErbB/HER receptors. Int J Radiat Oncol Biol Phys 58: 903-913, 2004

32. Gabillard JC, Ulisse S, Baldini E, et al: Aurora-C interacts with and phosphorylates the transforming acidic coiled-coil 1 protein. Biochem Biophys Res Commun 408: 647-653, 2011.

33. Knuefermann C, Lu Y, Liu B, et al: HER2/PI-3K/Akt activation leads to a multidrug resistance in human breast adenocarcinoma cells. Oncogene 22: 3205-3212, 2003. 